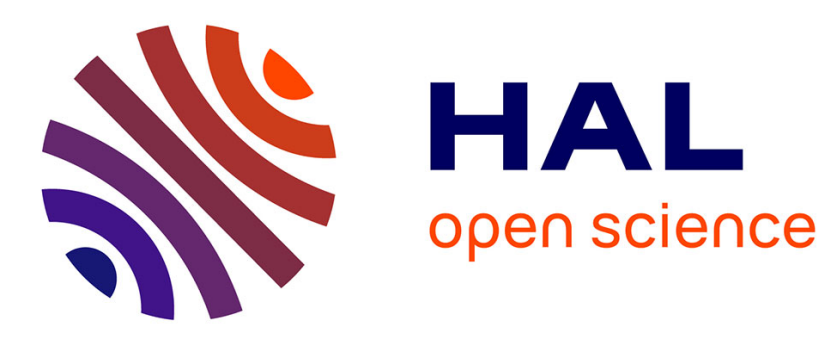

\title{
When will zeros of time-delay systems cross imaginary axis?
}

\author{
Jie Chen, P. Fu, Silviu-Iulian Niculescu
}

\section{To cite this version:}

Jie Chen, P. Fu, Silviu-Iulian Niculescu. When will zeros of time-delay systems cross imaginary axis?. 2007 9th European Control Conference, ECC 2007, Jul 2007, Kos, Greece. pp.5631-5638, 10.23919/ecc.2007.7068613 . hal-02272376

\section{HAL Id: hal-02272376 \\ https://hal.science/hal-02272376}

Submitted on 27 Aug 2019

HAL is a multi-disciplinary open access archive for the deposit and dissemination of scientific research documents, whether they are published or not. The documents may come from teaching and research institutions in France or abroad, or from public or private research centers.
L'archive ouverte pluridisciplinaire $\mathbf{H A L}$, est destinée au dépôt et à la diffusion de documents scientifiques de niveau recherche, publiés ou non, émanant des établissements d'enseignement et de recherche français ou étrangers, des laboratoires publics ou privés. 


\title{
When Will Zeros of Time-Delay Systems Cross Imaginary Axis?
}

\author{
Jie Chen, Peilin Fu and Silviu-Iulian Niculescu
}

\begin{abstract}
A time-delay system may or may not be stable for different periods of delay. When will then a delay system be stable or unstable, and for what ranges of delay? This paper attempts to answer these questions. We show that by finding a set of critical delay values, for which the system's characteristic quasipolynomial has zeros on the imaginary axis, it is possible to determine its stability in the full range of the delay parameter by characterizing the analytical behaviors of the zeros. This characterization is facilitated by an operator perturbation approach, which is both conceptually attractive and computationally efficient. The entire procedure, which first identifies the critical zeros on the imaginary axis and next determines whether the zeros cross the imaginary axis, requires only solving a generalized eigenvalue problem.
\end{abstract}

Index Terms - Time-delay, asymptotic stability, critical zeros, asymptotic behavior, matrix pencil.

\section{INTRODUCTION}

In this paper we study stability properties of linear timedelay systems. We consider specifically retarded systems with commensurate delays. For systems of this type, it is known that the stability problem can be tackled efficiently by computing the critical delay values that result in imaginary zeros of the system's characteristic quasipolynomial. This computation can be executed in a number of different ways, notably, by, e.g., solving a generalized eigenvalue problem [10], [16], [17]. Since the critical delay values form consecutive intervals and the zeros vary continuously with respect to the delay parameter, whether the system is stable over a specific delay interval can be determined by checking the stability corresponding to any fixed delay value within that interval, thus in principle, determining the system's stability over the entire range of delay values. One should note, nevertheless, that even for a fixed delay, the testing of stability for a time-delay system is not a simple task.

A more potent approach in accomplishing this goal is to analyze the asymptotic behavior of the critical zeros on the imaginary axis. The idea is rather simple. Specifically, at each critical delay value corresponding to a critical zero of the characteristic quasipolynomial, one may seek to determine whether the zero may cross the imaginary axis, from one half plane into another; for example, the system

This research is supported in part by CNSF/China, CNRS/France, and NSF/USA.

Jie Chen is with the Department of Electrical Engineering, University of California, Riverside, CA 92521 jchen@ee.ucr.edu

Peilin Fu is with the Department of Electrical Engineering, University of California, Riverside, CA 92521 pfudee.ucr.edu

Silviu-Iulian Niculescu is with Laboratoire des Signaux et Systèmes (L2S, UMR CNRS 8506), CNRS-Supélec, 3, rue Joliot Curie, 91192, Gifsur-Yvette, France. Silviu.Niculescullss.supelec.fr will become unstable if a critical zero enters the open righthalf plane, and otherwise will remain stable if all the critical zeros remain in the left-half plane. The essential problem in this approach, consequently, is to characterize the analytical properties of the imaginary zeros.

The problem alluded to above, known in the literature as the stability switch problem, has been studied for quite some time (see, e.g., [1], [5], [6], [12], [15], [18], [19]). Recently, the authors developed an operator perturbation approach [3] to facilitate this analysis, which appears both analytically attractive and computationally appealing. The approach seeks to reformulate the zero asymptotic analysis problem as one of eigenvalue perturbation [14], and it amounts to performing a first-order asymptotic expansion of the zeros around their critical values. Whether a critical zero will cross the imaginary axis can then be checked by computing the eigenvalues of a constant matrix. It is worth noting that the asymptotic expansion can be carried out in a rather efficient manner; in fact, it can be considered a direct consequence resulted from the computation of the critical delay values and the imaginary zeros.

This paper continues the work of [3] and gives a full development of the asymptotic analysis up to the second order. The results, along with the first-order analysis given in [3], thus provide a more complete tool which can aid in stability analysis when the first-order tests cease to be useful; this scenario arises when the first-order coefficient in the asymptotic series is imaginary, which renders the firstorder test inconclusive. Similar to their first-order counterparts, the second-order tests developed herein also require computing the eigenvalues of a constant matrix only.

The remainder of this paper is organized as follows. In Section 2, we first develop general eigenvalue perturbation results, which serve as our primary technical machinery. We then apply these results to time-delay systems; we first consider in Section 3 systems in the state-space form, and next in Section 4 systems described by differential-difference equations. Conditions are derived for characterizing whether a critical zero will cross the imaginary axis. Section 5 presents an illustrative example and Section 6 concludes the paper.

We use the following notation throughout the paper. Let $\mathbb{R}$ be the set of real numbers, $\mathbb{C}$ the set of complex numbers, and $\mathbb{R}_{+}$the set of nonnegative real numbers. Denote the open right half plane by $\mathbb{C}_{+}:=\{s: \Re(s)>0\}$, the closed right half plane by $\overline{\mathbb{C}}_{+}$, and the imaginary axis by $\partial \mathbb{C}_{+}$. Similarly, denote the open unit disc by $\mathbb{D}$, the unit circle by $\partial \mathbb{D}$, and the closed exterior of the unit disc by $\mathbb{D}^{c}$. 
For a matrix $A$, denote its spectrum by $\lambda(A)$, and the ith eigenvalue by $\lambda_{i}(A)$. For a matrix pair $(A, B)$, denote the set of all its generalized eigenvalues by $\sigma(A, B)$, i.e.,

$$
\sigma(A, B):=\{\lambda \in \mathbb{C}: \operatorname{det}(A-\lambda B)=0\} .
$$

The operation $A \oplus B$ denotes the Kronecker sum, and $A \otimes B$ the Kronecker product, of the matrices $A$ and $B$.

\section{PREliminary Results}

This section introduces the operator perturbation theory for matrix eigenvalue problems. We shall first give an account of the first-order results developed in [3], and subsequently perform a second-order analysis. The development is based on the classical work by Kato [14] and the authors' own extension in [3].

\section{A. First-Order Analysis}

Consider a matrix operator $T(x)$ of a real variable $x$. Suppose that in the neighborhood of $x=0$, the perturbed operator $T(x)$ is holomorphic, or equivalently, can be expanded into the power series,

$$
T(x)=T(0)+x T^{\prime}(0)+x^{2} T^{\prime \prime}(0)+\cdots .
$$

It is known that near the origin, any semisimple eigenvalue of $T(x)$, namely the semisimple root of the characteristic equation

$$
\operatorname{det}(T(x)-\xi I)=0
$$

is an analytic functions of $x$ and can be expressed as a power series in $x$; here by a semisimple eigenvalue, we mean a repeated but diagonalizable eigenvalue. With no loss of generality, let $\lambda^{(0)}$ be ordered as the first eigenvalue of $T(0)$ with multiplicity $m$. Then $T(0)$ can be decomposed as

$$
T(0)=Q \Sigma R=\left[\begin{array}{ll}
Q_{1} & Q_{2}
\end{array}\right]\left[\begin{array}{cc}
\Sigma_{1} & 0 \\
0 & \Sigma_{2}
\end{array}\right]\left[\begin{array}{l}
R_{1} \\
R_{2}
\end{array}\right],
$$

where $\Sigma_{1}$ is diagonal with diagonal entries as $\lambda^{(0)}, R=$ $Q^{-1}=\left[\begin{array}{lll}r_{1}^{T} & \cdots & r_{n}^{T}\end{array}\right]^{T}$, and $Q=\left[\begin{array}{lll}q_{1} & \cdots & q_{n}\end{array}\right]$ consist of the eigenvectors of $T(0)$. The following lemma gives the asymptotic series for the eigenvalues of $T(x)$ originating from $\lambda^{(0)}$.

Lemma 1 [3] Let $\lambda^{(0)}$ be a semisimple eigenvalue of $T(0)$ with multiplicity $m$. Then the corresponding eigenvalues of $T(x)$ are analytic in $x$ and have the form

$$
\mu_{i}(x)=\lambda^{(0)}+\lambda_{i}^{(1)} x+o\left(x^{2}\right), \quad i=1, \ldots, m,
$$

where $\lambda_{i}^{(1)}, i=1, \ldots, m$, are the eigenvalues of $R_{1} T^{\prime}(0) Q_{1}$.

Consider next the case that $\lambda^{(0)}$ is not a semisimple but repeated eigenvalue of $T(0)$ with multiplicity $m$. In this case, $T(0)$ admits a Jordan decomposition in which $\Sigma$ is block diagonal with diagonal Jordan blocks, and $Q_{1}$ consists of the generalized eigenvectors associated with $\lambda^{(0)}$. In particular,

$$
\Sigma_{1}=\left[\begin{array}{cccc}
\lambda^{(0)} & 1 & \cdots & 0 \\
0 & \ddots & \ddots & 0 \\
\vdots & & \ddots & 1 \\
0 & \cdots & \cdots & \lambda^{(0)}
\end{array}\right] .
$$

The eigenvalue of $T(x)$ can no longer be expanded in the form of (4), but instead as a Puiseux series.

Lemma 2 [3] Let $\lambda^{(0)}$ be a non-semisimple eigenvalue of $T(0)$ with multiplicity $m$. Then the corresponding eigenvalues of $T(x)$ have the form

$$
\mu_{i}(x)=\lambda^{(0)}+\left(\gamma_{i}^{(1)}\right)^{\frac{1}{m}} x^{\frac{1}{m}}+\cdots, \quad i=1, \ldots, m,
$$

where $\gamma_{i}^{(1)}=r_{m} T^{\prime}(0) q_{1}$.

As a consequence, Lemma 1 and Lemma 2 provide a complete first-order characterization of the asymptotic expressions for the eigenvalues of $T(x)$ near the origin.

\section{B. Second-Order Asymptotic Expansion}

The first-order asymptotic series introduced in the preceding subsection can be further developed to include terms of higher orders. In this section we derive formulas for computing the coefficients of the asymptotic series up to the second order.

Define the operator-valued function

$$
\Upsilon(\xi)=(T(0)-\xi I)^{-1},
$$

which is known as the resolvent of $T(0)$. It is obvious that the singularities of $\Upsilon(\xi)$ are the eigenvalues of $T(0)$. Let $\lambda^{(0)}$ be an eigenvalue of $T(0)$. Then $\Upsilon(\xi)$ can be expanded as a Laurent series at $\xi=\lambda^{(0)}$, that is,

$$
\begin{aligned}
\Upsilon(\xi) & =-\left(\xi-\lambda^{(0)}\right)^{-1} P-\sum_{n=1}^{\infty}\left(\xi-\lambda^{(0)}\right)^{-n-1} D_{n} \\
& +\sum_{n=0}^{\infty}\left(\xi-\lambda^{(0)}\right)^{n} S_{n+1},
\end{aligned}
$$

where $P, D_{n}$ and $S_{n+1}$ are corresponding coefficient matrices. Evidently, the matrix $P$, known as the eigenprojection for $\lambda^{(0)}$, can be found as

$$
P=-\frac{1}{2 \pi j} \oint_{\Gamma} \Upsilon(\xi) d \xi=\frac{1}{2 \pi j} \oint_{\Gamma}(\xi I-T(0))^{-1} d \xi,
$$

where $\Gamma$ is a positively-oriented closed contour enclosing $\lambda^{(0)}$ but no other eigenvalues of $T(0)$. It was found in [3] that

$$
P=Q_{1} R_{1}
$$

The holomorphic part in the Laurent expansion is called the reduced resolvent of $T(0)$ with respect to the eigenvalue $\lambda^{(0)}$, denoted as

$$
S(\xi)=\sum_{n=0}^{\infty}\left(\xi-\lambda^{(0)}\right)^{n} S_{n+1}
$$


Let $S=S\left(\lambda^{(0)}\right)$, namely the value of the reduced resolvent of $T(0)$ at $\xi=\lambda^{(0)}$. Then it is obvious that

$$
S=S_{1}=\frac{1}{2 \pi j} \oint_{\Gamma} \frac{\Upsilon(\xi)}{\xi-\lambda^{(0)}} d \xi
$$

Lemma 3 For any matrix $T(0)$ decomposed in the form of (3), where $\Sigma_{1}$ is in Jordan form with diagonal entries as $\lambda^{(0)}$, the reduced resolvent at $\xi=\lambda^{(0)}$ is equal to

$S=Q\left[\begin{array}{cc}0 & 0 \\ 0 & \left(\Sigma_{2}-\lambda^{(0)} I\right)^{-1}\end{array}\right] R=Q_{2}\left(\Sigma_{2}-\lambda^{(0)} I\right)^{-1} R_{2}$.

The following Lemma given in [14] provides the result on the second order perturbation of $T(x)$ when all the eigenvalues of $T(0)$ are semisimple.

Lemma 4 [14] Let $\lambda^{(0)}$ be a semisimple eigenvalue of $T(0)$, $\lambda_{i}^{(1)}$ be a semisimple eigenvalue of $P T^{\prime}(0) P$ with the eigenprojection $P_{i}^{(1)}$, that is

$$
P_{i}^{(1)}=\oint_{\Gamma_{i}}\left(\xi I-P T^{\prime}(0) P\right)^{-1} d \xi,
$$

where $\Gamma_{i}$ is a positively-oriented closed contour enclosing $\lambda_{i}^{(1)}$ but no other eigenvalues of $P T^{\prime}(0) P$. Then $T(x)$ has $d=\operatorname{dim} P_{i}^{(1)}$ repeated eigenvalues of the form

$$
\mu_{i p}(x)=\lambda^{(0)}+x \lambda_{i}^{(1)}+x^{2} \mu_{i p}^{(2)}+o\left(x^{2}\right), \quad p=1, \cdots, d,
$$

where $\mu_{i p}^{(2)}$ are the repeated eigenvalues of

$$
P_{i}^{(1)} T^{(2)} P_{i}^{(1)}=P_{i}^{(1)}\left(T^{\prime \prime}(0)-T^{\prime}(0) S T^{\prime}(0)\right) P_{i}^{(1)} .
$$

The eigenvalues of $P_{i}^{(1)} T^{(2)} P_{i}^{(1)}$ can be computed in a manner similar to that in the first-order analysis.

Lemma 5 Let $\lambda_{i}^{(1)}$ be an eigenvalue of $P T^{\prime}(0) P$. Let also $T(0)$ be decomposed as in (3), and $R_{1} T^{\prime}(0) Q_{1}$ be decomposed as

$$
\begin{aligned}
R_{1} T^{\prime} & (0) Q_{1} \\
& =Q^{(2)} \Sigma^{(2)} R^{(2)} \\
& =\left[\begin{array}{ll}
Q_{1}^{(2)} & Q_{2}^{(2)}
\end{array}\right]\left[\begin{array}{cc}
\Sigma_{1}^{(2)} & 0 \\
0 & \Sigma_{2}^{(2)}
\end{array}\right]\left[\begin{array}{c}
R_{1}^{(2)} \\
R_{2}^{(2)}
\end{array}\right]
\end{aligned}
$$

where $\Sigma_{1}^{(2)}$ is the Jordan block corresponding to the eigenvalue $\lambda_{i}^{(1)}$. Then,

$$
P_{i}^{(1)}=Q_{1} Q_{1}^{(2)} R_{1}^{(2)} R_{1},
$$

and the eigenvalues of $P_{i}^{(1)} T^{(2)} P_{i}^{(1)}$ are those of the matrix $R^{(2)} R_{1} T^{(2)} Q_{1} Q_{1}^{(2)}$.

We note that when $\lambda_{i}^{(1)}$ is a simple eigenvalue, then $P_{i}^{(1)}=q_{1} r_{1}$.

Finally, in the case that $\lambda_{1}^{(i)}$ is a repeated non-semisimple eigenvalue of $P T^{\prime}(0) P$, we can state a result similar to Lemma 2. We state this result without proof.

Lemma 6 Let $\lambda^{(0)}$ be a semisimple eigenvalue of $T(0)$, and $\lambda_{i}^{(1)}$ be a non-semisimple eigenvalue of $P T^{\prime}(0) P$ with multiplicity $d$. Then the $d$ corresponding eigenvalues of $T(x)$ can be expanded in the form

$$
\begin{array}{r}
\mu_{i}(x)=\lambda^{(0)}+x \lambda_{i}^{(1)}+\left(\tilde{r}_{d} T^{(2)} \tilde{q}_{1}\right)^{1 / d} x^{1+\frac{1}{d}}+\cdots \\
i=1, \ldots, d, \quad
\end{array}
$$

where $\tilde{r}_{d}$ and $\tilde{q}_{1}$ are the dth right and the first left eigenvectors of $P T^{\prime}(0) P$, respectively.

\section{State-Space Models}

In this section we consider linear time-delay systems described by the state-space equation

$$
\dot{x}(t)=A_{0} x(t)+\sum_{k=1}^{q} A_{k} x(t-k \tau), \quad \tau \geq 0,
$$

where $\tau$ is the delay parameter and $A_{k} \in \mathbb{R}^{n \times n}$ are the given system matrices. The characteristic quasipolynomial associated with this system is given by

$$
p\left(s, e^{-\tau s}\right)=\operatorname{det}\left(s I-\sum_{k=0}^{q} A_{k} e^{-k \tau s}\right) .
$$

For a fixed $\tau \geq 0$, the system is asymptotically stable if and only if all the zeros of the quasipolynomial $p\left(s, e^{-\tau s}\right)$ lie in $\mathbb{C}^{-}$(see, e.g., [10]).

The critical delay values and the critical zeros of the above system can be computed in the following way. Define the matrices

$$
\begin{aligned}
U= & {\left[\begin{array}{ccccc}
I & 0 & \cdots & 0 & 0 \\
0 & I & \cdots & 0 & 0 \\
& & \ddots & & \\
0 & 0 & \cdots & I & 0 \\
0 & 0 & \cdots & 0 & B_{2 q}
\end{array}\right] } \\
V= & {\left[\begin{array}{ccccc}
0 & I & 0 & \cdots & 0 \\
0 & 0 & I & \cdots & 0 \\
0 & 0 & 0 & \cdots & I \\
-B_{0} & -B_{1} & -B_{2} & \cdots & -B_{2 q-1}
\end{array}\right], }
\end{aligned}
$$

where $B_{m} \in \mathbb{R}^{n^{2} \times n^{2}}, m=0,1, \cdots, 2 q$ are given by

$$
B_{q-m}=I \otimes A_{m}^{T}, \quad B_{q}=A_{0} \oplus A_{0}^{T}, \quad B_{q+m}=A_{m} \otimes I .
$$

The following lemma, obtained in [4] (see also [9]), shows that the critical delay values and zeros can be determined by solving a generalized eigenvalue problem.

Lemma 7 The characteristic quasipolynomial $p\left(s, e^{-\tau s}\right)$ has a critical zero on the imaginary axis if and only if the following conditions are satisfied:

(i) $\sigma(V, U) \cap \partial \mathbb{D} \neq \emptyset$;

(ii) For some $z_{i} \in \sigma(V, U) \cap \partial \mathbb{D}$,

$$
\sigma\left(\sum_{k=0}^{q} A_{k} z_{i}^{k}\right) \cap j \mathbb{R}_{+} \neq \emptyset .
$$


The imaginary number $j \omega_{i} \in \sigma\left(\sum_{k=0}^{q} A_{k} z_{i}^{k}\right)$, where $\omega_{i}>$ 0 , is a critical zero. The corresponding critical delay forms the set

$$
\mathcal{T}\left(\omega_{i}\right)=\left\{\frac{\log \left(\overline{z_{i}}\right)}{j \omega_{i}}+\frac{2 \pi \ell}{\omega_{i}}>0, \ell=1,2, \cdots\right\},
$$

where $\log (\cdot)$ represents the Cauchy principal value and $\overline{(.)}$ the complex conjugate.

Note that while $\mathcal{T}\left(\omega_{i}\right)$ is a countable set consisting of infinitely many elements, the number of critical zeros is finite. In addition, since the zeros of $p\left(s, e^{-\tau s}\right)$ are conjugate symmetric, it suffices to consider only the critical zeros with $\omega_{i}>0$. The entire range of delay values can be partitioned into intervals $\left(\tau_{i}, \tau_{i+1}\right)$, and the stability is invariant in each of such intervals.

Let $\tau^{*}$ and $j \omega^{*}, \omega^{*} \in \mathbb{R}_{+}, \omega^{*} \neq 0$ be a pair of critical delay and critical zero of $p\left(s, e^{-\tau s}\right)$, i.e., $p\left(j \omega^{*}, e^{-j \omega^{*} \tau^{*}}\right)=$ 0 . The asymptotic behavior of the critical imaginary zero $j \omega^{*}$ can be examined by casting the problem into one of eigenvalue perturbation. Indeed, introduce a new real variable $x=\tau-\tau^{*}$, and define

$$
T(x):=\sum_{k=0}^{q}\left(A_{k} e^{-j \omega^{*} k \tau^{*}}\right) e^{-j \omega^{*} k x} .
$$

Clearly, $T(x)$ is holomorphic. Furthermore, since

$$
\operatorname{det}\left(T(0)-j \omega^{*} I\right)=p\left(j \omega^{*}, e^{-j \omega^{*} \tau^{*}}\right)=0,
$$

where

$$
T(0)=\sum_{k=0}^{q} A_{k} e^{-j \omega^{*} k \tau^{*}},
$$

$j \omega^{*}$ is an eigenvalue of $T(0)$. Without loss of generality, let $j \omega^{*}$ be ordered as the first eigenvalue of $T(0)$, with multiplicity $m$. Let also $T(0)$ be partitioned as in (3). Then, the following asymptotic expansion can be obtained.

Theorem 1 Let $j \omega^{*}$ be a semisimple eigenvalue of $T(0)$ with multiplicity $m$, and let $\lambda_{i}^{(1)}$ be a semisimple eigenvalue of $P T^{\prime}(0) P$ with multiplicity $d$. Then for any $\tau$ sufficiently close to $\tau^{*}$, the characteristic zeros corresponding to $j \omega^{*}$ can be expanded into the power series

$$
j \omega^{*}+\lambda_{i}^{(1)}\left(\tau-\tau^{*}\right)+\mu_{i p}^{(2)}\left(\tau-\tau^{*}\right)^{2}+o\left(\left(\tau-\tau^{*}\right)^{3}\right),
$$

with

$$
\begin{array}{r}
\lambda_{i}^{(1)}=\lambda_{i}\left(R_{1} T^{\prime}(0) Q_{1}\right), \\
i=1,2, \cdots, m, \\
\mu_{i p}^{(2)}=\lambda_{p}\left[R_{1}^{(2)} R_{1}\left(T^{\prime \prime}(0)-T^{\prime}(0) S T^{\prime}(0)\right) Q_{1} Q_{1}^{(2)}\right], \\
p=1,2, \cdots, d,
\end{array}
$$

where

$$
\begin{aligned}
T^{\prime}(0) & =-\sum_{k=1}^{q} j k \omega^{*} A_{k} e^{-j \omega^{*} k \tau^{*}}, \\
T^{\prime \prime}(0) & =-\sum_{k=1}^{q}\left(\omega^{*} k\right)^{2} A_{k} e^{-j \omega^{*} k \tau^{*}},
\end{aligned}
$$

and $S$ is given in Lemma 3.

(i) For $\tau$ sufficiently close to $\tau^{*}$ but $\tau>\tau^{*}$, there are $m$ repeated characteristic zeros entering the right-half plane (or the left-half plane) if the following eigenvalues satisfy the condition

$$
\operatorname{Re}\left\{\lambda_{i}\left(R_{1} T^{\prime}(0) Q_{1}\right)\right\}>0(<0) .
$$

(ii) If

$$
\operatorname{Re}\left\{\lambda_{i}\left(R_{1} T^{\prime}(0) Q_{1}\right)\right\}=0,, \quad i=1,2, \cdots, m,
$$

then for $\tau$ sufficiently close to $\tau^{*}$ but $\tau>\tau^{*}$, there are $d$ repeated characteristic zeros entering the right-half plane (or the left half plane) if the eigenvalues $\mu_{i p}^{(2)}$ satisfy the condition

$$
\begin{gathered}
\operatorname{Re}\left\{\lambda_{p}\left[R_{1}^{(2)} R_{1}\left(T^{\prime \prime}(0)-T^{\prime}(0) S T^{\prime}(0)\right) Q_{1} Q_{1}^{(2)}\right]\right\} \\
>0(<0) .
\end{gathered}
$$

Thus, upon finding the critical delays and critical zeros, the additional computation for the asymptotic expansion given in Theorem 1 requires only computing the eigenvalues in the expressions of $\lambda_{i}^{(1)}$ and $\mu_{i p}^{(2)}$. In particular, when $j \omega^{*}$ is a simple critical zero, this computation can be further simplified. The following corollary is an immediate consequence of Theorem 1 .

Corollary 1 Let $j \omega^{*}$ be a simple eigenvalue of $T(0)$. Then for any $\tau$ sufficiently close to $\tau^{*}$ but $\tau>\tau^{*}, j \omega^{*}$ enters the right-half plane (or the left-half plane) if

$$
\operatorname{Re}\left\{r_{1} T^{\prime}(0) q_{1}\right\}>0 \quad(<0),
$$

where $q_{1}$ and $r_{1}$ are the right and left eigenvectors associated with $j \omega^{*}$. Additionally, if

$$
\operatorname{Re}\left\{r_{1}\left(T^{\prime}(0)\right) q_{1}\right\}=0,
$$

then for any $\tau$ sufficiently close to $\tau^{*}$ but $\tau>\tau^{*}, j \omega^{*}$ enters the right-half plane (or the left half plane) if

$$
\operatorname{Re}\left\{r_{1}\left(T^{\prime \prime}(0)-T^{\prime}(0) S T^{\prime}(0)\right) q_{1}\right\}>0 \quad(<0),
$$

The next two results concern the cases that $j \omega^{*}$ is not a semisimple but repeated eigenvalue, or that $\lambda_{i}^{(1)}$ is such an eigenvalue.

Theorem 2 Let $j \omega^{*}$ be a repeated eigenvalue of $T(0)$ with multiplicity $m$. Suppose that $j \omega^{*}$ is not semisimple. Then for any $\tau$ sufficiently close to $\tau^{*}$ but $\tau>\tau^{*}$, the $m$ characteristic zeros corresponding to $j \omega^{*}$ can be expanded into the Puiseux series

$$
j \omega^{*}+\left\{-r_{m} T^{\prime}(0) q_{1}\right\}^{\frac{1}{m}}\left(\tau-\tau^{*}\right)^{\frac{1}{m}}+\cdots,
$$

Hence, for $k=0,1, \cdots, m-1$, the kth branch of the eigenvalue enters the right half plane (or the left half plane) if

$$
\cos \left(\frac{2 k \pi+\pi+\theta}{m}\right)>0 \quad(<0),
$$

where $\theta \in[0,2 \pi]$ is the phase angle of $r_{m} T^{\prime}(0) q_{1}$. 
Theorem 3 Let $j \omega^{*}$ be a semisimple eigenvalue of $T(0)$ with multiplicity $m$ and $\lambda_{i}^{(1)}=\lambda_{i}\left(R_{1} T^{\prime}(0) Q_{1}\right)$ be a repeated eigenvalue of $P T^{\prime}(0) P$ with multiplicity $d$. Suppose that $\lambda_{i}^{(1)}$ is not semisimple. Then for any $\tau$ sufficiently close to $\tau^{*}$ but $\tau>\tau^{*}$, the characteristic zeros corresponding to $j \omega^{*}$ can be expanded by the Puiseux series

$j \omega^{*}+\lambda_{i}^{(1)}\left(\tau-\tau^{*}\right)+\left(-\tilde{r}_{d} T^{(2)} \tilde{q}_{1}\right)^{\frac{1}{d}}\left(\tau-\tau^{*}\right)^{1+\frac{1}{d}}+\cdots$,

where

$$
T^{(2)}=R_{1}^{(2)} R_{1}\left(T^{\prime \prime}(0)-T^{\prime}(0) S T^{\prime}(0)\right) Q_{1} Q_{1}^{(2)},
$$

and $\tilde{r}_{d}, \tilde{q}_{1}$ are the dth right and the first left eigenvectors of $P T^{\prime}(0) P$. Hence, if

$$
\operatorname{Re}\left\{\lambda_{i}\left(R_{1} T^{\prime}(0) Q_{1}\right)\right\}=0,
$$

then for $k=0,1, \cdots, d-1$, the kth branch of the eigenvalue enters the right half plane (or the left half plane) if

$$
\cos \left(\frac{2 k \pi+\pi+\tilde{\theta}}{d}\right)>0 \quad(<0)
$$

where $\tilde{\theta} \in[0,2 \pi]$ is the phase angle of $\tilde{r}_{m} T^{(2)} \tilde{q}_{1}$.

Theorem 2 and Theorem 3 reveal a fundamental difference between the asymptotic behaviors of a semisimple eigenvalue and one that is not. Take Theorem 2 for example, for a repeated eigenvalue that is not semisimple, the result shows that, precluding the case that

$$
\operatorname{Re}\left\{r_{m} T^{\prime}(0) q_{1}\right\}=0,
$$

whether the zero will enter the right-half plane is solely determined by the multiplicity of the zero. In this case, the branches of the zero loci will almost generically enter the right-half plane when the multiplicity is greater than two. Note that in the degenerate case $m=1$, Theorem 2 also reduces to Corollary 1.

\section{Differential-Difference Equation Model}

Alternatively, we also consider the differential-difference equation

$$
y^{(n)}(t)+\sum_{i=0}^{n-1} \sum_{k=0}^{q} a_{k i} y^{(i)}(t-k \tau)=0, \quad \tau \geq 0 .
$$

Systems described by (19) can be represented equivalently by the quasipolynomial

$$
a\left(s, e^{-\tau s}\right)=\sum_{k=0}^{q} a_{k}(s) e^{-k \tau s}, \quad \tau \geq 0,
$$

where

$a_{0}(s)=s^{n}+\sum_{i=0}^{n-1} a_{0 i} s^{i}, \quad a_{k}(s) \sum_{i=0}^{n-1} a_{k i} s^{i}, \quad k=1, \cdots, q$.
We note that a similar generalized eigenvalue-based solution (see, e.g., [4]) exists for the critical zeros $j \omega^{*}$ of $a\left(s, e^{-\tau s}\right)$ at the critical delay value $\tau^{*}$, at which $a\left(j \omega^{*}, e^{-j \omega^{*} \tau^{*}}\right)=$ 0 . This result is available from [4], [9].

Lemma 8 Define $H_{n}:=0, T_{n}:=I$, and for $i=$ $0,, 1, \cdots, n-1$,

$$
\begin{aligned}
T_{i}:= & {\left[\begin{array}{cccc}
a_{0 i} & 0 & \cdots & 0 \\
a_{1 i} & a_{0 i} & \cdots & 0 \\
\vdots & \ddots & \ddots & \vdots \\
a_{q-1, i} & a_{q-2, i} & \cdots & a_{0 i}
\end{array}\right], } \\
H_{i}:= & {\left[\begin{array}{cccc}
a_{q i} & a_{q-1, i} & \cdots & a_{1 i} \\
0 & a_{q i} & \cdots & a_{2 i} \\
\vdots & \ddots & \ddots & \vdots \\
0 & 0 & \cdots & a_{q i}
\end{array}\right] }
\end{aligned}
$$

Define further

$$
\begin{gathered}
P_{i}:=\left[\begin{array}{ccc}
(j)^{i} T_{i} & (j)^{i} H_{i} \\
(-j)^{i} H_{i}^{T} & (-j)^{i} T_{i}^{T}
\end{array}\right], \quad i=0,1, \cdots, n, \\
P:=\left[\begin{array}{cccc}
0 & I & \cdots & 0 \\
\vdots & \vdots & \ddots & \vdots \\
0 & 0 & \cdots & I \\
-P_{n}^{-1} P_{0} & -P_{n}^{-1} P_{1} & \cdots & -P_{n}^{-1} P_{n-1}
\end{array}\right] . \\
F(s):=\left[\begin{array}{cccc}
0 & 1 & \cdots & 0 \\
\vdots & \vdots & \ddots & \vdots \\
0 & 0 & \cdots & 1 \\
-a_{0}(s) & -a_{1}(s) & \cdots & -a_{q-1}(s)
\end{array}\right], \\
G(s):=\operatorname{diag}\left(1 \quad \cdots \quad 1 \quad a_{q}(s)\right) .
\end{gathered}
$$

The quasipolynomial $a\left(s, e^{-\tau s}\right)$ has a critical zero on the imaginary axis if and only if the following conditions are satisfied:

(i) $\sigma(P) \cap \partial \mathbb{R}_{+} \neq \emptyset$ and $\sigma(P) \cap \partial \mathbb{R}_{+} \neq\{0\}$;

(ii) For some $\omega_{i} \in \sigma(P) \cap \partial \mathbb{R}_{+}, \sigma\left(F\left(j \omega_{i}\right), G\left(j \omega_{i}\right)\right) \cap$ $\partial \mathbb{D}=\emptyset$.

The imaginary number $j \omega_{i} \in \sigma(P)$, where $\omega_{i}>0$, is a critical zero. The corresponding critical delay forms the set

$$
\mathcal{T}\left(\omega_{i}\right)=\left\{\frac{\log \left(\overline{z_{i}}\right)}{j \omega_{i}}+\frac{2 \pi \ell}{\omega_{i}}>0, \ell=1,2, \cdots\right\},
$$

where $z_{i} \in \sigma\left(F\left(j \omega_{i}\right), G\left(j \omega_{i}\right)\right)$.

It is clear that Theorem 1-Theorem 3 can be directly applied to determine the asymptotic behavior of the critical 
zero $j \omega^{*}$, by making use of the realization

$$
\begin{aligned}
A_{0}= & {\left[\begin{array}{cccc}
0 & 1 & \cdots & 0 \\
\vdots & \vdots & \ddots & \vdots \\
0 & 0 & \cdots & 1 \\
-a_{00} & -a_{01} & \cdots & -a_{0, n-1}
\end{array}\right] } \\
A_{k}= & {\left[\begin{array}{cccc}
0 & 0 & \cdots & 0 \\
\vdots & \vdots & \ddots & \vdots \\
0 & 0 & \cdots & 0 \\
-a_{k 0} & -a_{k 1} & \cdots & -a_{k, n-1}
\end{array}\right], k>0 }
\end{aligned}
$$

Additionally, with the specific structures of this realization, it is not difficult to derive that the eigenvalues of $T(0)$ are also the roots of quasipolynomial $a\left(s, e^{-j \omega^{*} \tau^{*}}\right)$. This makes it possible to state the results directly in terms of the quasipolynomial $a\left(s, e^{-\tau s}\right)$. We first show that with a realization given by the above companion form, $T(0)$ cannot have semisimple repeated eigenvalues.

Lemma 9 Any companion matrix

$$
A=\left[\begin{array}{cccc}
0 & 1 & \cdots & 0 \\
\vdots & \vdots & \ddots & \vdots \\
0 & 0 & \cdots & 1 \\
-\alpha_{0} & -\alpha_{1} & \cdots & -\alpha_{n-1}
\end{array}\right]
$$

cannot have semisimple repeated eigenvalues.

The implication of Lemma 9 is rather clear. With the above realization of $A_{k}$, the matrix $T(0)$ is in the companion form with

$$
\alpha_{i}=\sum_{k=0}^{q} a_{k i} e^{-j k \omega^{*} \tau^{*}}, \quad i=0,1 \cdots, n-1 .
$$

As such, when applying the results in Section III, one can preclude the case that $T(0)$ has semisimple repeated eigenvalues; instead, the critical zero $j \omega^{*}$ is either a simple eigenvalue, or a repeated, non-semisimple eigenvalue of $T(0)$. Theorem 4 and Corollary 2 given below describe the asymptotic behavior of imaginary poles in these two cases, in terms of $a\left(s, e^{-\tau s}\right)$.

Theorem 4 Let $j \omega^{*}$ be a repeated zero of a $\left(s, e^{-j \omega^{*} \tau^{*}}\right)$ with multiplicity $m$. Then for any $\tau$ sufficiently close to $\tau^{*}$ but $\tau>\tau^{*}$, the $m$ zeros corresponding to $j \omega^{*}$ can be expanded into the Puiseux series

$j \omega^{*}+\left(-\left.m ! \frac{\left.\frac{d a\left(j \omega^{*}, e^{-j \omega^{*} \tau}\right)}{d \tau}\right|_{\tau=\tau^{*}}}{\frac{d^{m} a\left(s, e^{-j \omega^{*} \tau^{*}}\right)}{d s^{m}}}\right|_{s=j \omega^{*}}\right)^{\frac{1}{m}}\left(\tau-\tau^{*}\right)^{\frac{1}{m}}+\cdots$,

If $j \omega^{*}$ is a simple zero of $a\left(s, e^{-j \omega^{*} \tau^{*}}\right)$, the following corollary is an immediate consequence of Theorem 4.

Corollary 2 Let $j \omega^{*}$ be a simple zero of a $\left(s, e^{-j \omega^{*} \tau^{*}}\right)$. Then for any $\tau$ sufficiently close to $\tau^{*}$ but $\tau>\tau^{*}, j \omega^{*}$ enters the right-half plane (or the left-half plane) if

$$
\operatorname{Re}\left\{\frac{\left.\frac{d a\left(j \omega^{*}, e^{-j \omega^{*} \tau}\right)}{d \tau}\right|_{\tau=\tau^{*}}}{\left.\frac{d a\left(s, e^{-j \omega^{*} \tau^{*}}\right)}{d s}\right|_{s=j \omega^{*}}}\right\}<0 \quad(>0) .
$$

It is useful to note that in the case of a simple critical zero, results similar to Corollary 2 have been reported in [1], [5], [6], [12], [19], while without much technical deliberation. Our derivation herein thus provides an independent, rigorous justification to some of the previously available results.

Theorem 4 and Corollary 2 correspond to the firstorder analysis of the quasipolynomial. When these results become inapplicable, the following second-order result can be employed.

Theorem 5 Let $j \omega^{*}$ be a simple zero of a $\left(s, e^{-j \omega^{*} \tau^{*}}\right)$. If

$$
\operatorname{Re}\left\{\frac{\left.\frac{d a\left(j \omega^{*}, e^{-j \omega^{*} \tau}\right)}{d \tau}\right|_{\tau=\tau^{*}}}{\left.\frac{d a\left(s, e^{-j \omega^{*} \tau^{*}}\right)}{d s}\right|_{s=j \omega^{*}}}\right\}=0,
$$

then for any $\tau$ sufficiently close to $\tau^{*}$ but $\tau>\tau^{*}, j \omega^{*}$ enters the right-half plane (or the left-half plane) if

$$
\begin{aligned}
& \operatorname{Re}\left\{\frac{\frac{d^{2} a\left(j \omega^{*}, e^{-j \omega^{*} \tau}\right)}{d \tau^{2}}}{\frac{d a\left(s, e^{-j \omega^{*} \tau^{*}}\right)}{d s}}\right. \\
& \left.-\left(\frac{\frac{d a\left(j \omega^{*}, e^{-j \omega^{*} \tau}\right)}{d \tau}}{\frac{d a\left(s, e^{-j \omega^{*} \tau^{*}}\right)}{d s}}\right)\left(\frac{\frac{\partial^{2} a\left(s, e^{-j \omega^{*} \tau}\right)}{\partial s \partial \tau}}{\frac{d a\left(s, e^{-j \omega^{*} \tau^{*}}\right)}{d s}}\right)\right\}<0 \quad(>0)
\end{aligned}
$$

at $s=j \omega^{*}, \tau=\tau^{*}$.

\section{An Illustrative EXAmple}

In this section we use an example to demonstrate the second-order zero-crossing conditions.

Example Consider the following second-order delay system $^{1}$ [12] (see also [11]):

$$
\left[\begin{array}{l}
\dot{x}_{1}(t) \\
\dot{x}_{2}(t)
\end{array}\right]=\left[\begin{array}{cc}
0 & 1 \\
-1 & -1
\end{array}\right]\left[\begin{array}{l}
x_{1}(t) \\
x_{2}(t)
\end{array}\right]+\left[\begin{array}{cc}
0 & 0 \\
0 & -1
\end{array}\right]\left[\begin{array}{l}
x_{1}(t-\tau) \\
x_{2}(t-\tau)
\end{array}\right] .
$$

The corresponding characteristic quasipolynomial is given by

$$
p\left(s, e^{-\tau s}\right)=s^{2}+s+s e^{-s \tau}+1 .
$$

By a direct application of Lemma 7 or Lemma 8, a pair of critical zero and delay are found to be $j \omega^{*}=j$ and $\tau^{*}=\pi$.

Following the state-space computations in Theorem 1 and Corollary 1 , we first find

$$
T(0)=\left[\begin{array}{cc}
0 & 1 \\
-1 & -1
\end{array}\right]+\left[\begin{array}{cc}
0 & 0 \\
0 & -1
\end{array}\right] e^{-j \pi}=\left[\begin{array}{cc}
0 & 1 \\
-1 & 0
\end{array}\right],
$$

which can be decomposed as

$$
T(0)=\left[\begin{array}{cc}
1 & 1 \\
j & -j
\end{array}\right]\left[\begin{array}{cc}
j & 0 \\
0 & -j
\end{array}\right]\left[\begin{array}{cc}
1 / 2 & -(1 / 2) j \\
1 / 2 & (1 / 2) j
\end{array}\right] .
$$

${ }^{1}$ Note that the state-space form of this system was incorrectly given in [12]. 
Furthermore,

$$
T^{\prime}(0)=\left[\begin{array}{cc}
0 & 0 \\
0 & -j
\end{array}\right], \quad T^{\prime \prime}(0)=\left[\begin{array}{cc}
0 & 0 \\
0 & -1
\end{array}\right] .
$$

It follows instantly that $r_{1} T^{\prime}(0) q_{1}=-(1 / 2) j$. As such, it is necessary to invoke the second-order condition. For this purpose, we find

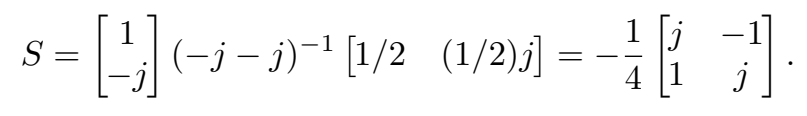

A trivial calculation shows that

$$
r_{1}\left(T^{\prime \prime}(0)-T^{\prime}(0) S T^{\prime}(0)\right) q_{1}=-\frac{1}{2}\left(1+\frac{1}{4} j\right) .
$$

This means that the zeros $\pm j$ enter the left-half plane. Since the system is stable for $\tau \in[0, \pi)$, we conclude that the critical zeros $\pm j$ do not cross the imaginary axis.

We may also use Corollary 2 and Theorem 5 to check the behavior of the imaginary zero $j \omega^{*}=j$. In this vein, we first compute

$$
\begin{aligned}
& \left.\frac{d p\left(j \omega^{*}, e^{-j \omega^{*} \tau}\right)}{d \tau}\right|_{\tau=\tau^{*}}=\left.\left(-\left(j \omega^{*}\right)^{2} e^{-j \omega^{*} \tau}\right)\right|_{\tau=\pi}=-1, \\
& \left.\frac{d p\left(s, e^{-j \omega^{*} \tau^{*}}\right)}{d s}\right|_{s=j \omega^{*}}=\left.\left(2 s+1+e^{-j \omega^{*} \tau^{*}}\right)\right|_{s=j}=2 j .
\end{aligned}
$$

This gives rise to

$$
\frac{\left.\frac{d p\left(j \omega^{*}, e^{-j \omega^{*} \tau}\right)}{d \tau}\right|_{\tau=\tau^{*}}}{\left.\frac{d p\left(s, e^{-j \omega^{*} \tau^{*}}\right)}{d s}\right|_{s=j \omega^{*}}}=\frac{1}{2} j,
$$

and hence Corollary 2 is rendered inconclusive. To proceed to the second-order condition, we find

$$
\begin{aligned}
\left.\frac{d^{2} p\left(j \omega^{*}, e^{-j \omega^{*} \tau}\right)}{d \tau^{2}}\right|_{\tau=\tau^{*}} & =\left.\left(\left(j \omega^{*}\right)^{3} e^{-j \omega^{*} \tau}\right)\right|_{\tau=\pi}=j, \\
\left.\frac{\partial^{2} p\left(s, e^{-j \omega^{*} \tau}\right)}{\partial s \partial \tau}\right|_{s=j \omega^{*}, \tau=\tau^{*}} & =\left.\left(-j \omega^{*} e^{-j \omega^{*} \tau}\right)\right|_{\tau=\pi}=j .
\end{aligned}
$$

It then follows that

$$
\begin{aligned}
& \left(\frac{\frac{d^{2} p\left(j \omega^{*}, e^{-j \omega^{*} \tau}\right)}{d \tau^{2}}}{\frac{d p\left(s, e^{-j \omega^{*} \tau^{*}}\right)}{d s}}\right) \\
& -\left.\left(\frac{\frac{d p\left(j \omega^{*}, e^{-j \omega^{*} \tau}\right)}{d \tau}}{\frac{d p\left(s, e^{-j \omega^{*} \tau^{*}}\right)}{d s}}\right)\left(\frac{\frac{\partial^{2} p\left(s, e^{-j \omega^{*} \tau}\right)}{\partial s \partial \tau}}{\frac{d p\left(s, e^{-j \omega *} \tau^{*}\right)}{d s}}\right)\right|_{s=j \omega^{*}, \tau=\tau^{*}} \\
& =\frac{1}{2}-\frac{1}{4} j,
\end{aligned}
$$

which, according to Theorem 5, indicates that the imaginary zeros $\pm j$ enter the left half plane, resulting in the same conclusion based on the state-space computation. Figure 1 confirms indeed that the zeros only "touch" the imaginary axis, but do not cross it.

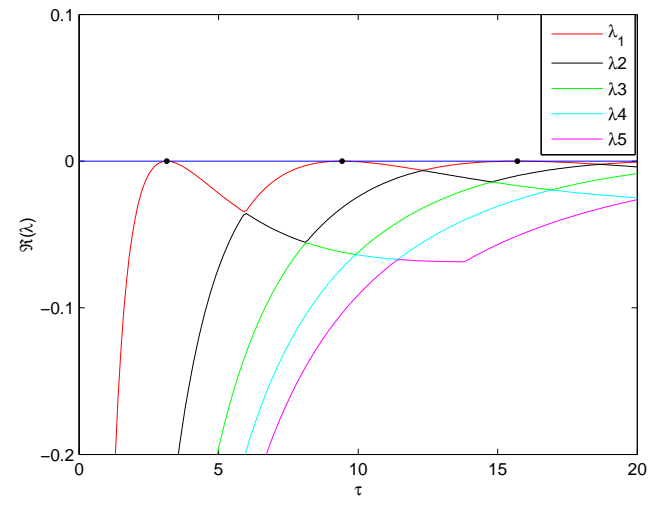

Fig. 1. Eigenvalues as functions of the delay $\tau$

\section{CONCLUding Remarks}

In this paper we have studied the asymptotic behavior of the critical characteristic zeros of linear time-delay systems with commensurate delays, for systems posed both in statespace form and as a quasipolynomial. We have shown that in both cases the asymptotic behavior of the critical zeros on the imaginary axis can be characterized in a simple manner, either by computing the eigenvalues of a constant matrix, or by computing the derivatives of the quasipolynomial. We demonstrated that when the first-order analysis ceases to be conclusive, the second-order condition can be used to effect.

\section{REFERENCES}

[1] F.G. Boese, Stability with respect to the delay: On the paper of K.L. Cooke and P. van den Driessche, J. Math. Anal.Appl., vol. 228, 1998, pp 293-321.

[2] C.T. Chen, Linear System Theorem and Design, CBS Colledge Publishing, New York; 1984.

[3] J. Chen, P. Fu and S.I. Niculescu, Asymptotic behavior of imaginary zeros of linear systems with commensurate delays, in Proceedings of the 45th IEEE Conference on Decision and Control, San Diego, CA, 2006, pp 1375-1380.

[4] J. Chen, C. Gu and C.N. Nett, A new method for computing delay margins for stability of linear delay systems, Syst. \& Contr. Lett., vol. 26, 1995, pp 101-117.

[5] K.L. Cooke and Z. Grossman, Discrete delay, distributed delay and stability switches, J. Math. Anal. Appl., vol. 86, 1982, pp 592-627.

[6] K.L. Cooke and P. Van den Driessche, On zeroes of some transcendental equations, Funkcialaj Ekvacioj, vol. 29, 1986, pp 77-90.

[7] A.S. Deif, Advanced Matrix Theory for Scientists and Engineers, Abacus Press, England; 1982.

[8] P. Freitas, Dealy-induced instabilities in gyroscopic systems, SIAM J. Contr. Optimiz., vol. 39, 2000, pp 196-207.

[9] P. Fu, S.I. Niculescu, and J. Chen, Stability of linear neutral timedelay systems: exact conditions via matrix pencil solutions, in Proceedings of 2005 American Control Conference, Portland, OR, 2005, pp 4259-4264.

[10] K. Gu, V.L. Kharitnov and J. Chen, Stability of Time-Delay Systems, Birkhauser, Boston; 2003.

[11] A. Gosiewski and A.W. Olbrot, The effects of feedback delays on the performance of multivariable linear control systems. IEEE Trans. Automat. Contr., AC-25, 1980, pp 729-735.

[12] D. Hertz, E.I. Jury and E. Zeheb, Simplified analytical stability test for systems with commensurate time delays, IEE Proc. Contr. Theory \& Appl., Pt. D, 131, 1984, pp 52-56.

[13] T. Kailath, Linear Systems, Prentice-Hall, Englewood Cliffs, NJ; 1980.

[14] T. Kato, Perturbation Theory for Linear Operations, Springer-Verlag, Berlin; 1995. 
[15] M.S. Lee and C.S. Hsu, On the $\tau$-decomposition method of stability analysis for retarded dynamical systems, SIAM J. Contr., vol. 7, 1969, pp. 249-259.

[16] S.I. Niculescu, Stability and hyperbolicity of linear systems with delayed state: A matrix pencil approach. IMA J. Math. Contr. Information, vol. 15, 1998, pp 331-347.

[17] S.I. Niculescu, Delay effects on stability. A robust control approach. Springer-Verlag: Heidelberg, LNCIS, vol. 269; 2001.

[18] N. Olgac, and R. Sipahi, An exact method for the stability analysis of time-delayed LTI systems, IEEE Trans. Automat. Contr., vol. 47, 2002, pp 793-797.

[19] K. Walton and J.E. Marshall, Direct method for TDS stability analysis, IEE Proc. Contr. Theory \& Appl., 134, Pt. D:2, 1987, pp 101-107. 